\title{
GEOMAGNETIC REVERSALS FROM IMPACTS ON THE EARTH
}

\author{
Richard A. Muller and Donald E. Morris
}

Lawrence Berkeley Laboratory, University of California, Berkeley, California 94720

\begin{abstract}
The impact of a large extraterrestrial object on the Earth can produce a geomagnetic reversal through the following mechanism: dust from the impact crater and soot from fires trigger a climate change and the beginning of a little ice age. The redistribution of water near the equator to ice at high latitudes alters the rotation rate of the crust and mantle of the Earth. If the sea-level change is sufficiently large $(>10$ meters) and rapid (in a few hundred years), then the velocity shear in the liquid core disrupts the convective cells that drive the dynamo. The new convective cells that subsequently form distort and tangle the previous field, reducing the dipole component near to zero while increasing the energy in multipole components. Eventually a dipole is rebuilt by dynamo action, and the event is seen either as a geomagnetic reversal or as an excursion. 'Sudden climate changes from other causes such as volcanic eruptions could also trigger reversals. This mechanism may not be the sole cause of geomagnetic reversals, but it can account for the rapid drop of the dipole component preceding a reversal, the predominance of multipole components during a transition, the associations of microtektites, temperature drops and extinctions with reversals, and the possible correlation between peaks in the geomagnetic reversal rate and the times of mass extinctions. The model may also account for the long-term changes in the average rate of reversals. We make several testable predictions.
\end{abstract}

\section{Introduction}

Geomagnetic reversals have proven to be a perplexing problem for geophysicists. Previous models to account for reversals assume that they occur spontaneously. A summary of reversal models has recently been published by J. A. Jacobs (1984). There is little direct evidence supporting previous models, and they do not explain several observed geophysical correlations, such as the occurrence of three of the four known tektite events at geomagnetic boundaries. We will discuss the correlations in more detail later in this paper.

We present here a description of the physical process by which the impact of a large extraterrestrial object can lead to a geomagnetic reversal. Although our model is somewhat speculative, it is based on assumptions considered plausible by workers in the relevant fields. The model readily explains the geophysical correlations, and accounts for the behavior of the Earth's field during a reversal.

\section{The Physical Process}

An asteroid or a comet nucleus $3 \mathrm{~km}$ in diameter moving at $25 \mathrm{~km} / \mathrm{sec}$ with respect to the Earth hits with a kinetic energy of $10^{29}$ ergs. The fireball from the impact lofts sufficient dust to darken the sky world-wide (Alvarez et al., 1980). Such dust, by serving as an intermediate absorber and radiator, interferes with the atmospheric greenhouse effect and causes a drop in the temperature of the continental land masses (Toon et al., 1982). The oceans, due to their high heat capacity, remain warm and continue to evaporate, while snow and ice build up on the continents and near the poles. Snow-covered land efficiently reflects sunlight, so the cool weather could persist, although complications from clouds

Copyright 1986 by the American Geophysical Union.

Paper number 6L7026.

0094-8276/86/006L-7026\$03.00 and storms make a detailed calculation to substantiate this assumption difficult. In this paper we do not investigate the effects of impacts on climate, but instead we discuss the effect of an impact-induced cooling on the Earth's dynamo.

The moment of inertia of the Earth is changed by the redistribution of tropical ocean water to ice at high latitudes, and this in turn alters the rotation rate of the Earth. Sudden sea level drops greater than 10 meters may have occurred 41 or more times during the last 65 million years (Vail and Hardenbol, 1979; Vail, personal communication) although the actual rate of fall of the sea level is too abrupt to be resolved, and it is not known whether the drops are coincident with impacts. (The present south-polar ice cap, should it melt, contains sufficient ice to raise the ocean levels by 50 to 100 meters.) If the transport occurs in a few centuries (too fast for isostatic adjustment of the moment of inertia of the Earth) then sufficient velocity shear is introduced between the Earth's mantle and its solid core to deform the convection cells in the liquid core of the Earth, as we will now show.

For simplicity, assume that all of the water to a depth-of 10 meters is deposited as snow and ice at high lattitudes. Prior to its removal (and neglecting details of geography) the shell of water had a moment of inertia $I_{w}=(2 / 3) M_{w} r_{e}{ }^{2}=$ $10^{39} \mathrm{gm} \mathrm{cm}^{2}$. The moment of inertia of the Earth (primarily due to the mantle) is $I_{e}=(2 / 5) M_{e} r_{e}^{2}=10^{45} \mathrm{gm-cm}^{2}$. From the conservation of angular momentum, the displacement of the water increases the angular velocity $\omega$ of the crust and mantle by $\Delta \omega / \omega=I_{w} / I_{m}=10^{-6}$. The increase in velocity at the bottom of the mantle is $\mathrm{v}=10^{-6} \mathrm{v}_{\mathrm{e}}=0.03 \mathrm{~cm} / \mathrm{sec}$. The angular shift between the core and the mantle is one radian after $t=(\Delta \omega)^{-1}=1.4 \times 10^{10} \mathrm{sec}=450$ years. Several small effects have been ignored, including the elastic rebound of the Earth (not be be confused with its slow isostatic adjustment through plastic deformation) and details of geography.

We wish to compare this $0.03 \mathrm{~cm} / \mathrm{sec}$ to the previously existing flow velocity $v_{d}$ in the liquid core. A rough estimate of $v_{d}$ can be obtained by assuming that the westward drift of the non-dipole component, about 0.18 degrees per year (Bullard et al., 1950), is due to transport of field by conductor moving with velocity $\mathrm{v}_{\mathrm{d}}$. This gives $\mathrm{v}_{\mathrm{d}} \approx 0.03 \mathrm{~cm} / \mathrm{sec}$. We can also estimate $v_{d}$ from theory. For dynamo action to take place, the velocity must be sufficiently high that the magnetic field is carried along by the moving fluid, yet low enough for significant diffusion; this consideration leads to a similar estimate of the convective velocity (Jacobs, 1984). We conclude that the new velocities produced by a ten meter sea-level-drop will be comparable to the preexisting velocities in the dynamo.

If the shearing motion cuts the existing magnetic field lines, the field will oppose the shear and cause angular momentum to be quickly transferred into the core. At the surface of the liquid core, the dipole magnetic field of the Earth is approximately 5 Gauss; estimates of the toroidal magnetic field in the core range from a few Gauss to a few hundred Gauss. The shear flow takes place approximately along the direction of the internal toroidal field, and the vorticity of the shear flow is along the lines of the poloidal field. Since the shearing surfaces contain the toroidal and dipole field lines, the shear is not accompanied by any change in the magnetic field energy. To the extent that the magnetic field is cylindrically symmetric, it does not resist the flow.

The non-dipole (higher multipole) poloidal components of the field will interact with the imposed shear flow. An imposed shear of $v=0.03 \mathrm{~cm} / \mathrm{sec}$ has kinetic energy density $1 / 2 p v^{2}$ comparable to the magnetic energy density $B^{2} /(8 \pi)=$ 
$6 \times 10^{-3} \mathrm{erg} / \mathrm{cm}^{3}$ of a 0.4 Gauss field. Note that the kinetic energy available to overcome the interacting magnetic field must be calculated in the rotating system, not in the inertial frame. If the radial component of the non-dipole magnetic field were much less than 0.4 Gauss, the outermost liquid core would be weakly coupled to the interior, and the shear would not be transmitted deep into the core; at the other extreme, if it were much greater than 0.4 Gauss, the torque would be rapidly coupled into the interior and to the solid core and the shear would not take place. The non-dipole field pattern is complex, with some regions of high radial field; the shear flow pattern can adjust itself to avoid these regions. Also, the flow can cut higher fields in some regions as long as the average field cut is small enough; since the fluid is effectively incompressible, and the entire kinetic energy of the shear flow is available. Extrapolation of the surface field to the core-mantle boundary (Bloxham and Gubbons, 1985) indicates that the non-dipole field over most of the core surface is sufficiently strong to couple angular momentum into the interior, yet weak enough to allow shearing of the liquid core.

Thus the shear flow transports material in the liquid core with a velocity comparable to that pre-existing in the core, but in a new direction that distorts the convective cells. In a few hundred years, a relative rotation of a radian takes place between the mantle and the solid core. As the shape of the cell is distorted, the temperature distribution no longer supports the previous flow field and the convective heat engine becomes ineffective. The convective velocities fall, and the source of kinetic energy for the dynamo is interrupted.

Buoyancy forces will dominate as a new flow pattern establishes itself, and the velocity field over much of the core will be substantially changed. The resulting flow will drag, distort and tangle the previous magnetic field into a configuration that initially has a scale size comparable to the convection cell size. The dipole will be destroyed in a time comparable to the several hundred year turn-over time of the new cells. This is much shorter than the $10^{4}-10^{5}$ yr free-decay time of the dynamo (the time for the dipole field to decay due to electrical resistance if there were no fluid velocity). The disappearance of the dipole component causes the magnitude of the magnetic field at the surface of the Earth to drop.

Eventually a new dynamo establishes itself. If the disruption of the previous dynamo was complete (as may be the case for a sufficiently large sea level drop), a geomagnetic reversal results in about half of the cases, and an "excursion" or "aborted reversal" in the other half.

\section{Discussion}

Our model for geomagnetic reversals is novel in that the changes in the convective flow pattern in the liquid core are driven by externally caused changes in mantle motion. In contrast, most previous explanations assume that they occur spontaneously, and thus must postulate that the convective flow pattern which sustains the dynamo is intrinsically unstable, an assumption that is not needed in our theory. Our model gives a natural explanation for the dominance of multipole components during a reversal, in contrast to spontaneous reversal models (Parker, 1969; Levy 1972) which require survival of the main dynamo in part of the liquid core during a spontaneous change in the convective flow pattern, in order to produce a reversal.

C. Doake (1977) suggested that the Earth's magnetic field could be disrupted by a change in rotation rate caused by a 100 meter change in ocean level at the onset or termination of an ice age. Although he did not propose a specific mechanism for the reversal, he did point out that previous models assumed reversals occurred spontaneously and therefore it was plausible that small perturbations could stimulate similar changes. He suggested that the change in the Earth rotation rate would "alter conditions at the core-mantle boundary and perturb the magnetic field or even cause a reversal." Doake also considered the possible resistance of the Earth's magnetic field to the velocity changes. He calculated the additional kinetic energy imparted to the core from the increased spin of the Earth, found it to be comparable to the total magnetic field energy, and concluded that the velocity changes would not be resisted by the field. Doake did the calculation in an inertial frame; in fact, only the much smaller kinetic energy calculated in the rotating frame is available to move the fluid against the resistance of the Earth's magnetic field. When the calculation is done in the rotating frame we find that the available kinetic energy is lower by several orders of magnitude than the value used by Doake. Nevertheless, the convective cells can still be disrupted since the cylindrically-symmetric components of the field do not effectively oppose the shear.

It is interesting to note how spin-up of the mantle actually takes place. As water from the relatively warm oceans continues to evaporate near the equator and begins to travel north and (south), the coriolis force causes the migration to turn into cyclones. These cyclones rotate in the proper directions (clock wise in the south, counter-clockwise in the north) to speed up the rotation of the Earth. The energy to drive this heat engine can be supplied by cooling the oceans less than $1^{\circ} \mathrm{C}$.

Our model depends on the transfer of water at low latitudes to non-floating ice near the poles. Floating ice does not change the moment of inertia of the oceans sufficiently to affect the dynamo. Thus the presence of land near the poles plays an important role in our mechanism.

A sudden rise in sea level will have a similarly disruptive effect on the geomagnetic field. If the West Antarctic ice shelf is truly unstable, as some speculate, then global warming (perhaps from increasing atmospheric $\mathrm{CO}_{2}$ ) may trigger its slide from the continent into the sea. If that happens we will have the opportunity to test our theory directly by watching the slow-down of the Earth's spin and the beginning of the turnoff of the geomagnetic field. It is also conceivable that a "nuclear winter" might cause a sudden fall in sea level sufficient to produce a geomagnetic reversal. We do not recommend that either of these experiments be carried out to test our prediction.

\section{Geophysical Evidence}

Our model can account for the observed rapid reduction in the intensity of the magnetic field several thousand years prior to a reversal, and the dominance of multipole components during the period of low dipole field. For a detailed review of the extensive data that show these effects, see Jacobs (1984). For recent high resolution results in sedimentary rock see Valet et al. (1986).

The model can also account for correlations observed between the following phenomena: (a) geomagnetic reversals, (b) crater formation or microtektite levels, and (c) changes in temperature and biota extinctions. A useful review has been compiled by Glass (1982). Glass et al. (1979) state: "three out of four known tektite strewnfields appear to be associated with reversals of the Earth's magnetic field. Thus serious consideration should be given to the possibility that reversals of the Earth's magnetic field can be triggered by large impact events. Extinctions and first appearances of marine micro-organisms appear to coincide with at least two of the known tektite events." Our model provides a causal mechanism to explain their observations. Note that we expect to see reversals at about half the impact horizons.

Krishnamurthy et al.(1986) found striking evidence of brief periods of cold climate precisely at three recent geomagnetic boundaries. They found sharp peaks in $C / N$ and ${ }^{12} \mathrm{C} /$ ${ }^{13} \mathrm{C}$ ratios in rapidly deposited lake sediments, implying short intervals of low temperature at $0.73 \mathrm{Myr}, 1.87 \mathrm{Myr}$, and 2.01 Myr, coincident with the Bruhnes/Matuyama boundary, the beginning of the Olduvai, and the end of the Reunion event; they presented no data for the end of the Olduvai. Their most detailed measurements are at the beginning of the Olduvai, where the $\mathrm{C} / \mathrm{N}$ ratio increased from a background level $\leq 4$ to a value of 20 in a peak less than 20,000 years in duration. The peak was also present in $\delta^{13} \mathrm{C}$ with $5 \%$ amplitude. Glass (1982) points out that the Czechoslovakian tektite event associated with the 14.7 Myr old Ries Crater (Shaw and 
Wasserburg, 1982) may be associated with a temperature drop indicated by ${ }^{18} \mathrm{O}$ studies (Shackleton and Kennett, 1975).

The most conspicuous horizon of biostratigraphic change within the last $2.43 \mathrm{Myr}$ is the Brunhes/Matuyama magnetic reversal boundary, at which the extinction and first appearance of several foraminiferal and radiolarial species have been found (Keany and Kennet, 1972). This level is closely associated with the Australasian microtektite layer (Glass et al., 1979). In addition, Hays (1971) pointed out that the beginning of the Jaramillo event marks the extinction of at least one species of Radiolaria. This event has been associated with the Ivory Coast microtektite layer (Glass et al., 1979).

Previously noted coincidences between mass extinctions and sea level regressions have led some paleontologists to speculate that the sea level regression caused the extinctions, although we see here that it is possible that the extinctions and regressions (as well as geomagnetic reversals) could have been caused by the same agent, the bolide impact. A timeseries analysis of the rate of geomagnetic reversals (Negi and Tiwari, 1983; Raup, 1985) has led to speculation that there is a 30 Myr periodicity in this rate similar to the periodicity seen in mass extinctions (Raup and Sepkoski, 1984) and in impact cratering (Alvarez and Muller, 1984; Rampino and Stothers, 1984) but the evidence is not compelling. We do not claim that impacts caused all sea level regressions, or that all impacts will be accompanied by such regressions. Other effects could certainly lower the sea surface, including changes in the luminosity of the sun, large volcanic eruptions, and even inherent instability in the climate of the Earth.

Vail and Hardenbol (1979) noted that there is no evidence for glaciation at the times of many of the sudden sea level regressions. It is possible that the buildup of a moderate icefield several tens of meters thick could have been missed, particularly if it occurred on Antarctica. It has been argued (Matthews and Poore 1980) that Antarctica could have had significant ice as early as 80 to $100 \mathrm{Myr}$ BP. Short periods of cold climate (as found in the data of Krishnamurthy et al., 1986), might provide little lasting evidence of glaciation.

There have been no geomagnetic reversals for the last 700,000 years, a time of extensive glaciation. Changes in sea level and glaciation during this period (Kennett, 1982) may have been relatively slow compared with the rapid changes needed to produce geomagnetic reversals according to our model. Small yet sudden sea-level changes during this period may have triggered excursions rather than reversals; the record of magnetic excursions is incomplete and ambiguous. Several possible excursions (Blake, Biwa I and Biwa II) have been reported in the period 400 to $100 \mathrm{kyr} \mathrm{BP}$, and may coincide (Jacobs, 1984) with times of more rapid change in ice volume inferred from ${ }^{18} \mathrm{O}$ measurements in deep sea cores (Hays et al., 1976). In the interval 100 to $20 \mathrm{kyr}$ years BP the average rate of sea level change appears to have been about 50 meters in $10^{4}$ years (Bloom et al., 1974). The Lake Mungo excursion (Barbetti and McElhinny, 1972), about $30 \mathrm{kyr}$ BP, is also associated with a brief period of apparently rapid cooling (Hays et al., 1976).

In the late Quaternary between 17,000 to 7,000 years BP the sea level rose about 70-100 meters (Dillon and Oldale, 1978). The average rate at which the sea level changed during this period is probably less than 10 meters per 1000 years. However, a short period of rapid cooling (determined from ${ }^{18} \mathrm{O} /{ }^{16} \mathrm{O}$ records) and glacier advance, and a nearly 10 meter fall in sea level (Fairbridge, 1977) took place at about the time of the so-called Gothenburg magnetic excursion dated between 13,750-12,350 yrs BP (Mörner and Lanser, 1975).

Suggestions have been made of correlations between the Earth's magnetic field and climate for the last few hundred years, but they are, at best, disputed (see Jacobs 1984). Our theory does not predict such correlations. Rather, we predict that abrupt changes in sea level will be followed in $10^{3}-10^{4}$ years by an excursion or reversal of the field. Unfortunately, the time scales of regressions and reversals have not yet been cross-correlated with sufficient accuracy $(<100 \mathrm{kyr})$ to directly confirm this prediction. Also, not enough is known about the
Earth's dynamo to allow a precise calculations of the minimum rate of sea level change necessary to trigger a reversal or excursion. If the convection fluid velocities in the core are substantially greater than the $0.03 \mathrm{~cm} / \mathrm{sec}$ that we assumed, then a greater and more rapid change in sea level is required to affect the dynamo.

If sudden sea-level drops (from impacts or volcanic eruptions) are the sole cause of reversals, then the long period during which no reversals occurred (roughly 80-120 Myr BP) might be explained by a warmer Earth climate during this period, when sea-levels were high and even large impacts could not trigger a sufficient cooling to build up continental ice. Sea-level was rising during this period (Vail et al., 1977), although high levels persisted after $80 \mathrm{Myr}$ BP. The gradual increase in the rate of reversals during the last $70 \mathrm{Myr}$ (to over 3 per Myr) suggests a correlation with the gradual cooling of the average ocean temperature in the Tertiary and Quaternary (Shackleton and Kennett, 1975) which could enable the more frequent but smaller impacts or eruptions to induce reversals.

Wetherill and Shoemaker (1982) estimated that impact craters on the Earth with diameter $\geq 10 \mathrm{~km}$ are formed at a rate of $9 \pm 4$ per million years. If a large fraction of reversals are caused by impacts, we must assume that such relatively small impacts can trigger the rapid onset of a small ice age, at least in the climatic conditions of the Tertiary and Quaternary. An interesting example is the $10.5 \mathrm{~km}$-diameter Bosumptwi Crater in Ghana, one of the most recent known impact craters of this size. This crater has been identified as the source of the Ivory Coast tektite strewn field (Shaw and Wasserburg, 1982) which is associated with the Jaramillo magnetic event (Glass et al. 1979). Such a small crater could not have ejected enough dust to extinguish sunlight by itself, however if the impact caused widespread fires (from radiant heat and hot rocks) then the soot created could conceivably have blocked the light and triggered the climate change. The importance of fires was made clear by W. Wolbach et al. (1985) who discovered extensive soot in Cretaceous/ Tertiary boundary clay.

An impressive example of the close temporal association of a geomagnetic reversal with an impact comes from the 24 km-diameter, 14.8 Myr old Ries crater in Germany. J. Pohl $(1977,1978)$ found that the suevite fallback breccias which were partially melted by the impact (and therefore recorded the Earth's field) show reversed polarity, while the first sediments in the crater show normal magnetization. This indicates that a geomagnetic reversal took place soon after the impact, probably within a few thousand years. Our model readily accounts for this observation. Pohl suggested that similar measurements should be made in other impact craters. The relatively recent El'gygytgyn $(3.5 \pm 0.5 \mathrm{Myr})$ and Zhamanshin $(0.75 \pm$ $0.06 \mathrm{Myr}$ ) craters in the U.S.S.R. are interesting candidates for measurement. Grieve (1982) lists 25 craters with diameters $\geq 10 \mathrm{~km}$ with ages less than 160 million years; in 6 of these the crater floors are exposed with only remnants of crater-fill preserved. A determination of the relative polarity of magnetization of the impact melt and first sediments in several craters could help verify or disprove the theory.

We predict a strong correlation between the times of sudden sea level regressions and geomagnetic reversals, provided that the times scales for these data can be cross-calibrated to $\pm 10^{5} \mathrm{yr}$. The published dates of many of the known regressions are rounded to the nearest half-million years.

The iridium layer found at the Cretaceous/Tertiary boundary was not coincident with a geomagnetic reversal (Alvarez et al., 1980), but it is not known whether there was an excursion at that time. Other impact-produced iridium layers should also be studied to see if they are coincident with reversals or excursions. During periods of warm climate large impacts were probably required to trigger reversals, so reversal horizons within such periods are most likely to yield detectable levels of iridium.

\section{Conclusions}

There is strong evidence that at least some geomagnetic reversals have been caused by impacts of large objects on the 
Earth. This evidence includes the creation and deposition of microtektites in close time coincidence with reversals, and the fact that at least one reversal took place between the creation of a crater and the accumulation of sediment in that crater. Other indirect evidence includes the observation at geomagnetic boundaries of sudden temperature changes and biological extinctions, and correlation of the periodicities of mass extinctions and increases in the rate of magnetic reversals.

We have described a physical process that accounts for these data by showing how impacts can lead to geomagnetic reversals. This process also explains the observed abrupt reduction of the magnetic field intensity prior to a reversal, and the dominance of multipole fields during a reversal. Because no complete analytical model of the dynamo within the Earth has yet been found, it was not possible to give a detailed mathematical model of a reversal. Indeed, several of the numbers that we have had to use are uncertain and model-dependent; therefore of necessity our argument was qualitative. In addition, we have had to assume the correctness of several recent observations and theories, e.g. that a large impact leads to global cooling, followed by a sudden drop in sea level. Although many experts believe these assumptions to be plausible, there is not yet a consensus that they are true.

Our model makes several testable predictions which offset these drawbacks. Correlations should exist between sudden sea-level drops and both geomagnetic reversals and excursions. The impact melt and the early sediment in large impact craters should give evidence that a reversal or excursion took place within a few thousand years after the impact. Finally, impact-caused iridium layers in sedimentary rock should be present at many reversal and excursion horizons.

Acknowledgments. We thank W. Alvarez, W. Press, R. Jeanloz, P. Vail, and B. Glass for helpful discussions, and E. M. Purcell for pointing out that the energy available to modify the dynamo must be calculated in the rotating reference frame. This work was supported in part by the U.S. Dept. of Energy, and in part by a fellowship from the MacArthur Foundation.

\section{References}

Alvarez, L. W., W. Alvarez, F. Asaro, \& H. V. Michel, Extra-terrestrial Cause for the Cretaceous-Tertiary Extinction, Science, 208, 1095-1108, 1980.

Alvarez, W., and R. Muller, Evidence from crater ages for periodic impacts on the Earth, Nature, 308, 718-720, 1984.

Barbetti, M. F. and M. W. McElhinny, Evidence of a geomagnetic excursion 30,000 yr BP, Nature, 239, 327, 1972.

Bloxham, J. and D. Gubbons, The secular variation of Earth's magnetic field, Nature, 317, 777-781, 1985.

Bloom, A. L. et al., Quaternary sea level fluctuations on a tectonic coast, Quat. Res., 4, 185-205, 1974.

Bullard, E. C., C. Freedman, H. Gellman and J. Nixon, The westward drift of the Earth's magnetic field, Phil. Trans. Roy. Soc. London, A 243, 67, 1950.

Dillon, W. P. and R. N. Oldale, Late quaternary sea-level curve: reinterpretation based on glaciotectonic influence, Geology, 6, 56-60, 1979.

Doake, C. S. M., A possible effect of ice ages on the Earth's magnetic field, Nature, 267, 415-417, 1977.

Fairbridge, R., Global climate change during the $13,500 \mathrm{BP}$ Gothenburg geomagnetic excursion, Nature 265, 430, 1977.

Glass, B. P., Possible correlations between tektite events and climatic changes, in Silver and Schultz (1982) pp 251-256.

Glass, B. P., M. B. Swincki, and P. A. Zwart, Australasian, Ivory Coast and North American tektite strewn-fields: Size, mass and correlation with geomagnetic reversals and other Earth events, Proc. 10th Lunar Planet. Sci. Conf., Houston (Pergamon press, New York, 1979) pp. 2535-2545.

Grieve, R. A. F., The record of impact on Earth: implications for a major Cretaceous/Tertiary impact event, in Silver and Schultz (1982) pp 25-37.

Hays, J., Faunal extinctions and reversals of the Earth's magnetic field, Geol. Soc. America Bull., 82, 2433, 1971.

Hays, J., J. Imbrie and N. Shackleton, Variations in the Earth's orbit: pacemaker of the ice ages, Science, 194, 1121, 1976.

Jacobs, J. A., Reversals of the Earth's magnetic field (Adam Hilger Ltd, Bristol, 1984).

Keany, J., J. P. Kennet, Pliocene - early Pleistocene paleoclimatic history recorded in Antartic-subantarctic deep-sea cores, Deep-sea Research, 19, 529, 1972.

Kennett, J. P., Marine Geology, (Prentice Hall, Englewood Cliffs, NJ, 1982), pp 268-273, 745-751.

Krishnamurthy, R., S. Bhattacharya and S. Kusumgar, Paleoclimatic changes deduced from ${ }^{13} \mathrm{C} /{ }^{12} \mathrm{C}$ and $\mathrm{C} / \mathrm{N}$ ratios of Karewa lake sediments, Nature, 323, 150-152, 1986.

Levy, E. H., Kinematic reversal schemes for the geomagnetic dipole, Astrophys. J., 171, 635-642, 1972.

Matthews, R., and R. Poore, Tertiary $\delta^{18} \mathrm{O}$ record and glacioeustatic sea-level fluctuations, Geology, 8, 501, 1980.

Mörner, N-A., and J. Lanser, Paleomagnetism in deep-sea core A179-15, Earth Planet. Sci. Lett., 26, 121, 1975.

Negi, J. G. and R. K. Tiwari, Matching long term periodicities of geomagnetic reversals and galactic motions of the solar system, Geophys. Res. Lett., 10, 713-716, 1983.

Parker, E. N., The occasional reversal of the geomagnetic field, Astrophys. J., 158, 815-827, 1969.

Pohl, J., Palaeomagnetische und gesteinsmagnetische untersuchungen an den kernen der forschungsbohrung Noerdlingen 1973, Geologica Bavarica, 75, 329-348, 1977.

Poh1, J., Evidence for the coincidence of a geomagnetic reversal with the Ries impact event, in Proceedings of the 41st Meeting of the Meteoritical Society, Sudbury, Canada, p. $600,1978$.

Rampino, M. and R. Stothers, Terrestrial mass extinctions, cometary impacts and the Sun's motion perpendicular to the galactic plane, Nature, 308, 709-712, 1984.

Raup, D. M., Magnetic reversals and mass extinctions, Nature, 314, 341-343, 1985; Rise and fall of periodicity, Nature, 317, 384-385, 1985.

Raup, D. M. and J. Sepkoski, Periodicity of extinctions in the geologic past, Proc. Nat. Acad. Sci., 81, 801-805, 1984.

Shackleton, N. and J. Kennett, Paleotemperature history of the Cenozoic and the initiation of Antarctic glaciation: oxygen and carbon isotope analyses in DSDP sites 277 , 279, and 2821, in Initial Reports of the Deep Sea Drilling Project, 29, 743-755 (U.S. Print. Off., Wash. D.C., 1975).

Shaw, H. F. and G. J. Wasserburg, Age and provenance of the target materials for tektites and possible impactites as inferred from Sm-Nd and $\mathrm{Rb}-\mathrm{Sr}$ systematics, Earth Planet. Sci. Lett., 60, 155-177, 1982.

Silver, L. and P. Schultz, editors, Geological implications of impacts of large asteroids and comets on the earth, Geol. Soc. America Special Paper 190, Boulder, Colorado, 1982.

Toon, B. et al., Evolution of an impact-generated dust cloud and its effects on the on the atmosphere, in Silver and Schultz (1982) pp 187-200.

Vail, P., et al., Seismic stratigraphy and global changes of sea level, in Seismic Stratigraphy-Applications to Hydrocarbon Exploration, Charles E. Payton ed., Memoir 26 of the Am. Assoc. of Pet. Geol., Tulsa, 1977, pp 49-212.

Vail, P.R. and J. Hardenbol, Sea-level changes during the tertiary, Oceanus, 22, 71, 1979.

Valet, J.-P., C. Lal, and P. Tucholka, High-resolution sedimentary record of a geomagnetic reversal, Nature, 322 , 27-32, 1986.

Wetherill, G., and E. Shoemaker, Collision of astronomically observable bodies with the Earth, in Silver and Schultz (1982) pp 1-13.

Wolbach, W. S., R. S. Lewis, and E. Anders, Cretaceous extinctions: evidence for wildfires and search for meteoritic material, Science, 230, 167, 1985.

(Received August 19, 1986;

Accepted September 22, 1986) 